\title{
Design, Characterization, and In Vivo Pharmacokinetics of Tacrolimus Proliposomes
}

\author{
Vijaykumar Nekkanti, ${ }^{1,3}$ Javier Rueda, ${ }^{2}$ Zhijun Wang, ${ }^{2}$ and Guru V Betageri ${ }^{1,2}$
}

Received 10 September 2015; accepted 30 September 2015; published online 19 October 2015

\begin{abstract}
The objective of this study was to develop proliposomal formulation for a poorly bioavailable drug, tacrolimus. Proliposomes were prepared by thin film hydration method using different lipids such as hydrogenated soy phosphatidylcholine (HEPC), soy phosphatidylcholine (SPC), distearyl phosphatidylcholine (DSPC), dimyristoylphosphatidylcholine (DMPC), and dimyristoylphosphatidylglycerol sodium (DMPG) and cholesterol in various ratios. Proliposomes were evaluated for particle size, zeta potential, in vitro drug release, in vitro permeability, and in vivo pharmacokinetics. In vitro drug release was carried out in purified water using USP type II dissolution apparatus. In vitro drug permeation was studied using parallel artificial membrane permeation assay (PAMPA) and everted rat intestinal perfusion techniques. In vivo pharmacokinetic studies were conducted in male Sprague-Dawley (SD) rats. Among the different formulations, proliposomes with drug/DSPC/cholesterol in the ratio of 1:2:0.5 demonstrated the desired particle size and zeta potential. Enhanced drug release was observed with proliposomes compared to pure tacrolimus in purified water after $1 \mathrm{~h}$. Tacrolimus permeability across PAMPA and everted rat intestinal perfusion models was significantly higher with proliposomes. The optimized formulation of proliposomes indicated a significant improvement in the rate and absorption of tacrolimus. Following a single oral administration, a relative bioavailability of $193.33 \%$ was achieved compared to pure tacrolimus suspension.
\end{abstract}

KEY WORDS: everted rat; oral bioavailability; PAMPA; pharmacokinetics; proliposomes; tacrolimus.

\section{INTRODUCTION}

The oral route is the most commonly preferred route of administration because of its convenience in dosing and patient compliance. However, drugs administered by oral route may often result in suboptimal therapeutic response because of the drug's poor solubility, inadequate permeation, and extensive first-pass effect. Proliposomes are reported as drug delivery carriers for enhancing the oral bioavailability of drugs with poor bioavailability (1-7). Proliposomes are dry, free flowing powders that can form multilamellar vesicles upon hydration. Liposomes can either be formed in vivo upon contact with physiological fluids or preformed by hydration with water before administration (8). Due to structural similarity between phospholipid bilayers and biological membranes, liposomes play an imperative role in facilitating the oral absorption of the poorly soluble drugs (9). The lipidbased systems following oral administration, especially the formulations with long chain fatty acids, promote the

\footnotetext{
${ }^{1}$ Graduate College of Biomedical Sciences, Western University of Health Sciences, 309 E. Second Street, Pomona, California 91766, USA.

${ }^{2}$ College of Pharmacy, Western University of Health Sciences, Pomona, California, USA.

${ }^{3}$ To whom correspondence should be addressed. (e-mail: vnekkanti@westernu.edu)
}

absorption of lipophilic drugs by simulating the formation of low-density lipoproteins and chylomicrons which are transported via the intestinal lymphatic system, circumventing the first-pass effect resulting in improved oral bioavailability (10). Proliposomes is an emerging platform technology for improving the oral delivery of drugs with poor bioavailability (11). In this study, we made an effort to improve the oral bioavailability of tacrolimus by formulating into proliposomes.

Tacrolimus is a potent immunosuppressive drug used in transplanted patients to minimize the risk of organ rejection. Tacrolimus is also a drug of choice for atopic dermatitis in reducing the side effects related with topical corticosteroids. Tacrolimus is classified as a class II drug according to the biopharmaceutical classification system (12). Tacrolimus exhibits poor aqueous solubility, P-gp efflux, and extensive presystemic metabolism resulting in low bioavailability following oral administration (13-16). The reported mean bioavailability is approximately $21 \%$ with large intersubject variability. The therapeutic application of tacrolimus is complex because of its narrow therapeutic index $(5-15 \mathrm{ng} / \mathrm{mL})$ and large inter/intrasubject variability in pharmacokinetic profile. The half-life of tacrolimus is 8.7 to $11.3 \mathrm{~h}$ and the mean time to achieve the peak plasma concentrations was reported as 1.5 to $2.0 \mathrm{~h}$ (17-19). Tacrolimus is a known P-gp and P450 3A4 (CYP3A4) substrate; therefore, any P-gp or CYP3A4 modulator can alter the pharmacokinetic properties of tacrolimus (20). 
Several formulation approaches have been reported to improve the oral bioavailability of tacrolimus. Most of these techniques have been focused on improving the solubility and absorption of tacrolimus from the GI tract or reducing P-gp efflux and CYP-450-mediated metabolism, with limited success. The reported approaches include prodrug (21), cyclodextrin complexes (22), solid dispersions $(23,24)$, liposomes $(25)$, self-microemulsifying drug delivery system (26-28), nanocapsules (29), micelles (30), nanosomes (31), microspheres (32), and nanoliposomes of dry powder inhaler (33). However, these investigational findings have not effectively translated into human clinical trials except solid dispersions. The commercial formulation (Prograf Capsules ${ }^{\circledR}$ ) developed using solid dispersion technology requires about $2 \mathrm{~h}$ for complete drug release and exhibits $25 \%$ bioavailability (17). Therefore, there is a requisite for a versatile drug delivery system that can resolve formulation issues associated with tacrolimus and improve its therapeutic efficacy. To the best of our knowledge, there is no study reported comparing the in vivo pharmacokinetic performance of tacrolimus drug product developed using proliposomal formulation technology. Our investigation was primarily focused on developing an improved formulation for tacrolimus using proliposomes that are capable of enhancing the oral bioavailability of tacrolimus by increasing the GI transport and avoiding the first-pass metabolism (34).

Lipids can promote the transport of drugs through the lymphatic route $(35,36)$. Many of the possible mechanisms of uptake include increasing transcellular absorption of the drug by increasing the membrane fluidity, enhancing the paracellular transport by opening the tight junction, and inhibiting the P-glycoprotein and cytochrome P450 to increase intracellular concentration and formation of lipoprotein/chylomicron by the lipid (37). Lipid-based systems are also known to improve and normalize the drug absorption which might be beneficial for a drug with narrow therapeutic window, e.g., tacrolimus. Therefore, proliposomes are evaluated for improving the pharmacokinetic performance of tacrolimus. In this study, the prepared proliposomes containing tacrolimus were measured for particle size, zeta potential, in vitro drug release, in vitro permeability, and in vivo pharmacokinetics.

\section{MATERIALS AND METHODS}

\section{Materials}

Tacrolimus was purchased from Tecoland Corporation (Irvine, CA, USA). Soy phosphatidylcholine (SPC), distearoyl-phosphatidylcholine (DSPC), hydrogenated soy phosphatidylcholine (HSPC), dimyristoylphosphatidylcholine (DMPC), and dimyristoylphosphatidylglycerol sodium (DMPG) were purchased from Avanti Polar Lipids (Alabaster, AL, USA); cholesterol and Krebs-Hensleit buffer $(\mathrm{pH}$ 7.4) were purchased from Sigma Aldrich (St. Louis, MO, USA); and 96-well plates were purchased from Millipore (Millipore, Billerica, USA). Cannulated Sprague-Dawley (SD) rats were purchased from Harlan Laboratories (Indianapolis, IN, USA). Blank rat plasma was purchased from Valley Biomedical (Winchester, VA, USA). Hard gelatin capsules (size 1) were purchased from Capsugel Inc (Morristown, NJ, USA), and all reagents used were of analytical grade.

\section{Preparation of Proliposomes}

Tacrolimus proliposomes were formulated using SPC, DSPC, DMPG, HSPC, DMPC, and cholesterol in various ratios. The formulation details and their respective compositions are summarized in Table I. Briefly, the required amounts of tacrolimus, phospholipid, and cholesterol were weighed and dissolved in ethyl alcohol. The solvent from the resultant solution was removed using a rotavapor (Buchi R-210, Buchi Corporation, New Castle, DE, USA) to obtain dry proliposomes (11). The proliposomal formulations were then passed through a sieve $(50 \mathrm{mesh} ; 300 \mu \mathrm{m})$ to obtain free flowing powders. The proliposomal formulations were filled into glass scintillation vials and stored at $4{ }^{\circ} \mathrm{C}$ for further studies. The prepared formulations were filled into size 1 , hard gelatin capsules for in vitro dissolution studies.

\section{Analysis of Tacrolimus}

Drug content, encapsulation efficiency, in vitro drug release, and in vitro permeation sample analysis was carried out by an HPLC analytical method developed using a Waters Alliance 2695 HPLC (Waters Corporation, Milford, MA, USA) instrument. Chromatographic separation of the samples was attained by Inertsil ODS-3, C18, $250 \times 4.6 \mathrm{~mm}, 5 \mu \mathrm{m}$ stainless steel column (GL Sciences Inc., Torrance, CA, USA). The mobile phase consists of acetonitrile and water. The mobile phase was pumped in a gradient fashion at $1 \mathrm{~mL} / \mathrm{min}$ with detection at $213 \mathrm{~nm}$. The injection volume was $20 \mu \mathrm{L}$ and the column temperature was maintained at $25^{\circ} \mathrm{C}$. A calibration curve was prepared in the concentration range of $0.25-50 \mu \mathrm{g} /$ $\mathrm{mL}$ in acetonitrile. The method has been validated for inter and intraday differences.

Table I. Formula Composition, Particle Size, and Zeta Potential of Proliposomal Formulations

\begin{tabular}{lllcrl}
\hline Formula code & Lipid used & Drug/lipid/cholesterol ratio & Mean vesicle size $(\mathrm{nm} \pm \mathrm{SD})$ & PDI $(\mathrm{mean} \pm \mathrm{SD})$ & Zeta potential $(\mathrm{mV} \pm \mathrm{SD})$ \\
\hline F-I & SPC & $1: 2: 0.5$ & $1070.6 \pm 64.4$ & $0.531 \pm 0.029$ & $-4.20 \pm 1.12$ \\
F-II & DSPC & $1: 2: 0.5$ & $858.2 \pm 49.1$ & $0.650 \pm 0.024$ & $-6.90 \pm 2.21$ \\
F-III & HSPC & $1: 2: 0.5$ & $2345.7 \pm 71.5$ & $0.514 \pm 0.018$ & $-1.05 \pm 0.85$ \\
F-IV & DMPC & $1: 2: 0.5$ & $1091.8 \pm 69.6$ & $0.621 \pm 0.021$ & $-3.29 \pm 1.33$ \\
F-V & DMPG & $1: 2: 0.5$ & $2465.4 \pm 78.3$ & $0.625 \pm 0.031$ & $-6.23 \pm 2.42$ \\
\hline
\end{tabular}

PDI polydispersity index, SPC soy phosphatidylcholine, DSPC distearyl phosphatidylcholine, HSPC hydrogenated soy phosphatidylcholine, DMPC dimyristoylphosphatidylcholine, $D M P G$ dimyristoylphosphatidylglycerol sodium 


\section{Characterization}

Particle Size

The average particle size of the prepared proliposomes was measured by dynamic light scattering (DLS) technique using a zeta-sizer (Nano ZS-90, Malvern Instruments, Malvern, Worcestershire, UK). All measurements $(n=3)$ were conducted at $25^{\circ} \mathrm{C}$, using a refractive index of 1.33 . Vesicle size of the prepared proliposomes was determined following hydration of the proliposomal formulations using purified water at $25^{\circ} \mathrm{C}$ under sonication for $10 \mathrm{~min}$ using a bath sonicator (Branson Ultrasonics, Danbury, CT, USA).

\section{Zeta Potential Measurement}

The zeta potential of tacrolimus proliposomes was measured using a Malvern Zeta-sizer (Malvern Instruments, UK). The electrophoretic mobility of the particles under an applied electric field is measured using laser interferometric technique. The proliposomal formulations were diluted to 100 times using purified water, and zeta potential was measured by placing the diluted samples in an electrophoretic cell.

\section{Encapsulation Efficiency}

Samples of proliposomal formulations were reconstituted with a solvent mixture of water and acetonitrile (90:10) at room temperature. The hydrated proliposomal formulations of known concentration were transferred to Centrifree ${ }^{\circledR}$ tubes (molecular weight cutoff of 30,000) (Merck Millipore Ltd., Cork, Ireland). The sample tubes were centrifuged at $2500 \mathrm{rpm}$ for $20 \mathrm{~min}$ at room temperature. The encapsulated tacrolimus liposomes remain on the surface of the filter membrane, and free drug was collected into the bottom tube of the filter (38). The separated phase was transferred to a $10-\mathrm{mL}$ flask, diluted to volume with ethanol, and analyzed using the HPLC method. The amount of tacrolimus in the separated phase represented the amount of free drug. The experiment was performed in triplicate. The encapsulation efficiency was determined by calculating the difference between the initial amount taken and the amount recovered in the aqueous phase using Eq. (1) (39).

$\%$ drug encapsulated

$$
=\frac{(\text { Total amount of drug added }- \text { free drug })}{(\text { Total amount of drug added })} \times 100
$$

\section{Differential Scanning Calorimetric Analysis}

Differential scanning calorimetric (DSC) analysis was conducted to investigate the solid-state properties of the selected formulation. DSC profiles of pure tacrolimus, optimized proliposomal formulation, and its physical mixture containing drug, phospholipid, and cholesterol were carried out using a DSC-823 (Mettler Toledo Instruments Columbus, $\mathrm{OH})$ connected to a RTE-140 (Neslab, Newington, NH) refrigeration unit. About 3 to $5 \mathrm{mg}$ of sample was placed in an aluminum pan and sealed. The experimental runs for each sample were set from 0 to $200^{\circ} \mathrm{C}$ at a ramping speed of $10^{\circ} \mathrm{C} / \mathrm{min}$, under a stream of nitrogen.

\section{Formation of Liposomes}

Cryogenic-transmission electron microscopic (cryoTEM) was used to study the formation of vesicles upon hydration with purified water. The liposomal dispersion was shaken for $5 \mathrm{~min}$ at room temperature to enable the formation of liposomes. A drop of the liposomal dispersion was placed on a sample grid (Formvar/carbon 300 mesh copper grids). The samples were then frozen using Vitrobot Mark IV (FEI Company, Eindhoven, Netherlands) (40). The prepared samples were observed under a cryo-TEM (TF20, FEI Company, Netherlands) at a magnification of 15,000 at $100 \mathrm{kV}$.

\section{In Vitro Dissolution Studies}

The release studies for the prepared formulations were conducted in USP Type II dissolution apparatus (Agilent 708DS, Santa Clara, CA, USA) connected to an autosampler (Agilent 850-DS, Santa Clara, CA, USA). The paddle speed was set at $75 \mathrm{rpm}$ and temperature of the media was adjusted to $37 \pm 0.2^{\circ} \mathrm{C}$. The dissolution medium used was $900 \mathrm{~mL}$ of purified water. Pure tacrolimus and proliposomal formulations equivalent to $5 \mathrm{mg}$ dose filled into size 1 hard gelatin capsules were tested for dissolution. Samples were collected at predetermined time intervals up to $1 \mathrm{~h}$, filtered using a 0.45 $\mu \mathrm{m}$ filter, and analyzed using HPLC. The percentage drug released from the formulations was estimated, and the average of the three measurements was used for analyzing the data.

\section{Parallel Artificial Membrane Permeation Assay Studies}

A 96-well filter plate was used for performing the parallel artificial membrane permeation assay (PAMPA) study. The filter plate assembly consists of two parts, a receiver pate and a donor plate. Five microliters of $1 \% w / v$ solution of lecithin in dodecane was added to each donor plate well to enable formation of an artificial membrane. The selected proliposomal formulation was diluted with purified water to obtain a known concentration of the drug $(1 \mathrm{mg} / \mathrm{mL})$. Pure drug suspension of equivalent strength to the formulation was prepared using $0.5 \%$ methyl cellulose solution. One hundred fifty microliters of the prepared formulation was added into the donor plate well and $300 \mu \mathrm{L}$ of phosphate buffer ( $\mathrm{pH}$ 7.4) was added in to each of the receptor plate well. The donor plate containing the sample was placed on the receptor cell plate carefully and incubated for $16 \mathrm{~h}$ at room temperature. Samples from the respective plates (donor and receptor) were collected at predetermined time intervals and analyzed using the HPLC method. The permeation of the drug across the membrane was estimated using Eq. (2) (2).

$$
P_{\mathrm{e}}=\frac{-\operatorname{In}\left[1-\frac{C_{\mathrm{A}}(t)}{C_{\mathrm{equilibrium}}}\right]}{A\left(\frac{1}{V_{\mathrm{D}}}+\frac{1}{V_{\mathrm{A}}}\right) t}
$$

Where $P_{\mathrm{e}}$ is the permeability in centimeters per second, $A$ is the effective filter area $\left(0.24 \mathrm{~cm}^{2}\right), V_{\mathrm{D}}$ is the well volume 
$(0.3 \mathrm{~mL})$ of the donor plate, $V_{\mathrm{A}}$ is the well volume $(0.2 \mathrm{~mL})$ of the receptor plate, $t$ is the incubation time $(\mathrm{s}), C_{\mathrm{A}}(t)$ is the amount of drug in the receptor plate well at time $t, C_{\mathrm{D}}(t)$ is the amount of drug in the donor plate well at time $t$, and

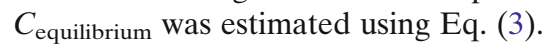

$C_{\text {equilibrium }}=\frac{\left[C_{\mathrm{D}}(t) * V_{\mathrm{D}}+C_{\mathrm{A}}(t) * V_{\mathrm{A}}\right]}{C_{\mathrm{A}}+V_{\mathrm{D}}}$

\section{Everted Rat Intestinal Perfusion Studies}

Everted rat intestinal perfusion studies (ex vivo) were conducted according to the method reported by Ravis and Feldman (41). The anesthetized nontreated SD rats were taken and insertion was made in the midline of the abdomen to remove the intestine. The first $15 \mathrm{~cm}$ of the intestine portion was discarded and the isolated intestine was cleaned using saline solution (cold) to discard its contents. The cleaned intestine was then everted using a glass rod. The everted intestine was cut into small portions of $6 \mathrm{~cm}$ long. Each portion of the intestine was mounted in the perfusion apparatus housed with two ports. One port was used for addition and removal of serosal fluid (42) and the second port was used to maintain continuous circulation of carbon dioxide $(5 \%)$ and oxygen $(95 \%)$ during the study. The perfusion apparatus was filled with the test solution, which served as the mucosal fluid (outer compartment) for the experiment. All the test solutions were prepared in Krebs-Hensleit buffer ( $\mathrm{pH}$ 7.4). Samples were withdrawn at regular time intervals for $1 \mathrm{~h}$. The collected samples were centrifuged at $1500 \mathrm{rpm}$ for $10 \mathrm{~min}$ and analyzed for tacrolimus content using the HPLC method.

\section{Permeability Analysis}

The absorption rate constant $\left(K_{\mathrm{a}}\right)$ of tacrolimus from pure drug suspension and proliposomes was estimated by plotting the slope of drug amount remaining versus time. The effective permeability coefficient $\left(P_{\text {eff }}\right)$ of tacrolimus across the membrane was estimated using Eq. (4).

$P_{\text {eff }(\text { rat })}=-\left[Q_{\text {in }} \cdot \operatorname{In}\left(C_{\text {in }} / C_{\text {out }(\text { corrected })}\right)\right] / A$

where $Q_{\text {in }}$ is the rate of the intestinal fluid $(0.2 \mathrm{~mL} / \mathrm{min})$, and $A$ is the intestinal surface area which is considered to be the area of a cylinder $(2 \pi r l)$ with the length $(l)$ and radius $(r)$ of $0.18 \mathrm{~cm} . C_{\text {in }}$ and $C_{\text {out }}$ are the concentrations at the inlet and corrected outlet, respectively. The $C_{\text {out (corrected) was measured }}$ using Eq. (5) (43).

$C_{\text {out }(\text { corrected })}=C_{\text {out }} \cdot\left(Q_{\text {out }} / Q_{\text {in }}\right)$

Where $Q_{\text {out }}$ is the estimated flow of the intestinal fluid $(\mathrm{mL} / \mathrm{min})$ at a specific time point. The enhancement ratio (ER) was estimated using Eq. (6) (44).

ER $=P_{\text {eff }}$ of formulation $/ P_{\text {eff }}$ of pure drug suspension

\section{Quantification of Tacrolimus in Plasma Using LC/MS/MS}

A stock solution of $1 \mathrm{mg} / \mathrm{mL}$ of tacrolimus was prepared by dissolving pure tacrolimus in methanol, and a series of working solutions in the concentrations of 31.25 , $62.5,250,500$, and $1000 \mathrm{ng} / \mathrm{mL}$ were prepared using methanol. The plasma samples for plotting the standard curves were spiked with these working standard solutions. Sirolimus was used as the internal standard (IS). A protein precipitation method was employed for the extraction of tacrolimus and IS. Briefly, the working standard and IS solutions were added to $100 \mu \mathrm{L}$ of plasma and mixed well. To the sample, $500 \mu \mathrm{L}$ of methanol was added and shaken vigorously using a vortex mixer, followed by centrifugation at $10,000 \mathrm{rpm}$ for $10 \mathrm{~min}$ at $4^{\circ} \mathrm{C}$. The organic supernatant was transferred and evaporated to dryness by flushing nitrogen at room temperature. The final residue was reconstituted using $100 \mu \mathrm{L}$ of methanol and $5 \mu \mathrm{L}$ was injected into the LC/MS/MS system for analysis.

The chromatographic separation was achieved using a Zorbax SB-C18 column (Agilent, Santa Clara, CA, USA, particle size $5 \mu \mathrm{m} ; 2.1 \times 150 \mathrm{~mm}$ ) protected with a guard column $(12.5 \times 2.1 \mathrm{~mm})$ at room temperature. The mobile phase consisted of acetonitrile and $0.1 \%$ formic acid in water (80:20). The flow rate used was $0.30 \mathrm{~mL} / \mathrm{min}$. The LC/MS/MS system (API 3200, Framingham, MA, USA) was used as the detector. The mass spectrometric conditions were as follows: gas 1, nitrogen (40 psi); gas 2, nitrogen (20 psi); ion spray voltage, $-4500 \mathrm{~V}$; ion source temperature, $450^{\circ} \mathrm{C}$; and curtain gas, nitrogen (25 psi). Multiple reaction monitoring (MRM) scanning in negative ion mode was used to monitor the transition of 802.3167.0 for tacrolimus and 958.4-166.9 for IS. Accuracy and precision within run and between the run was carried out using three different series at concentrations of $62.5,250$, and $1000 \mathrm{ng} / \mathrm{mL}$ of a mixture of tacrolimus and sirolimus.

\section{Pharmacokinetic Study}

Male SD rats with jugular vein cannulation (230$250 \mathrm{~g}$ body weight) were used for the pharmacokinetic study. The SD rats were distributed into two subgroups each containing five animals. Prior to the experiment, all animals were fasted for $18 \mathrm{~h}$ with free access to water. Group 1 animals were administered with $0.5 \mathrm{~mL}$ of pure tacrolimus suspension at a dose of $10 \mathrm{mg} / \mathrm{kg}$ with an oral gavage, and group 2 animals were administered with proliposomal formulation. Following oral administration, $0.3 \mathrm{~mL}$ of blood samples were collected from the cannula at $0.5,1,2,4,6$, and $24 \mathrm{~h}$ and transferred into a heparinized Eppendorf tube. A "zero" time sample was collected prior to administration of the dose. The collected samples were centrifuged at $13,000 \mathrm{rpm}$ for $8 \mathrm{~min}$ at $4^{\circ} \mathrm{C}$, and the supernatant plasma was collected and stored at $-80^{\circ} \mathrm{C}$ until analysis. Food was provided to the rats $4 \mathrm{~h}$ after the dosing. The protocol for animal study was approved by the IACUC, Western University of Health Sciences, Pomona, CA, USA. Animals were sacrificed after completion of the study according to the procedure defined in the IACUC protocol. 


\section{Statistical Analysis}

Statistical analysis of the drug release was conducted by one-way analysis of variance (ANOVA) and by evaluating the data using a model-independent method (45). The mean dissolution time (MDT) of the proliposomes was calculated and evaluated using ANOVA to investigate the statistical variance. The MDT values were estimated using Eq. (7).

$\operatorname{MDT}=\frac{\sum_{j=1}^{n} \widehat{t_{J}} \Delta M_{j}}{\sum_{j=1}^{n} \Delta M_{j}}$

Where $j$ represents the sample number, $n$ is the number of sampling time intervals, $t j$ is the time at midpoint between $t_{j}$ and $t_{j-1}$ (calculated by using the expression $\left(t_{j}+t_{j-1}\right) / 2$ ), and $\Delta M_{j}$ is the excess amount of drug released between $t_{j}$ and $t_{j-1}$. Similarly, the effective permeability values $\left(P_{\mathrm{e}}\right)$ of PAMPA and the permeability values $\left(P_{\text {eff }}\right)$ of rat intestine study were also analyzed using one-way ANOVA. A $P$ value less than 0.05 was considered statistically significant and the theoretical and estimated values of $F$ ( $F_{\text {critical }}$ and $\left.F_{\text {calculated }}\right)$ were compared for interpreting the results and to study the statistical variance. The in vitro dissolution profiles were analyzed for $f_{2}$ factor to measure the difference or similarity in the drug release profile (46).

\section{Formulation Stability}

Tacrolimus proliposomal formulation stability was evaluated by analyzing the physical appearance, particle size, and drug content upon storage at room temperature $\left(25^{\circ} \mathrm{C}\right)$ and refrigerated $\left(5 \pm 3^{\circ} \mathrm{C}\right)$ conditions for 3 months. The samples of proliposomes were analyzed at predetermined time intervals for particle size distribution and drug content.

\section{RESULTS AND DISCUSSION}

\section{Preparation of Proliposomes}

Proliposomes are novel drug carriers that can be used for improving the permeation and absorption of drugs with poor oral bioavailability (47). In order to study the effect of phospholipid composition on the physicochemical properties of liposomes, different phospholipids with varying acyl chain lengths, degree of saturation, and bilayer fluidity were evaluated for preparing the proliposomal formulations. Gregoriadis et al. in 1979 showed that inclusion of cholesterol in the liposomal formulation enhances the in vivo stability by increasing the membrane rigidity (48). This factor is of utmost importance as liposomes are unstable in the presence of acidic $\mathrm{pH}$, bile salts, and enzymes. Various formulations with drug/lipid/cholesterol in the ratios of 1:0.5:0.25, 1:1:0.25, $1: 2: 0.25$, and 1:2:0.5 were prepared and characterized for particle size, zeta potential, and encapsulation efficiency (data not shown). Among the evaluated compositions, the formulation with drug/lipid/cholesterol in the ratio of 1:2:0.5 has demonstrated the desired properties. Therefore, this ratio was selected for screening the formulations. The compositions of lipid, drug, and cholesterol selected for formula optimization are summarized in Table I.

\section{Characterization}

\section{Particle Size}

The particle size of proliposomal formulations is shown in Table I. The mean particle size of the liposomal formulations was in the range of $858 \pm 49.1$ to $2465 \pm 78.3 \mathrm{~nm}$. The liposomal formulation F-II containing drug/DSPC/cholesterol in the ratio of 1:2:0.5 showed the smallest particle size $(858 \pm 49.1 \mathrm{~nm})$ and the largest particle size was observed with formulation F$\mathrm{V}(2465 \pm 78.3 \mathrm{~nm})$ containing drug/DMPG/cholesterol in the ratio of 1:2:0.5. These results indicated that the size of vesicles formed differs based on the nature of the lipid used in the formulation. Packing of lipid in the liposome membranes has a major role on the physical membrane properties such as permeability, membrane elasticity, surface charge, and binding properties of proteins (49). The particle size range $(858 \pm 49.1$ to $2465 \pm 78.3 \mathrm{~nm}$ ) indicates that upon hydration, proliposomal formulations predominantly resulted in multilamellar liposomes. The advantages of multilamellar liposomes are that they exhibit high encapsulation efficiency for water-insoluble compounds and also are known to enhance the lymphatic transport of drugs (50). Polydispersity index (PDI) gives a measure of the width of particle size distribution (51). A PDI greater than 0.1 is considered to indicate a heterogeneous system. From Table I, it is evident that PDI of the particle size produced from the proliposomal formulation ranged from $0.514 \pm 0.018$ to $0.650 \pm 0.024$ indicating a polydisperse system.

\section{Zeta Potential}

Formulations having high zeta potential will oppose aggregation and remain stable due to the net charge on the dispersed particles (52). Zeta potential of the selected proliposomal formulations is summarized in Table I. The results indicate that formulation F-II containing tacrolimus/ DSPC/cholesterol has the highest zeta potential $(-6.90 \pm 2.21)$ as compared to the other formulations. The observed negative potential may be attributed to the negative charge on the polar head group of the phospholipid. The advantage of such negative charge is that they may prevent aggregation of vesicles and thus maintain a homogenous suspension (53).

\section{Encapsulation Efficiency}

The encapsulation efficiency of the selected formulations is shown in Fig. 1. The results envisage that encapsulation efficiency of the proliposomal formulations ranged between $44.8 \pm 8.4 \%$ and $85.8 \pm 7.2 \%$. The encapsulation efficiency is determined by the physicochemical properties of the drug, lipid composition, and amount of cholesterol used in the formulation. The results indicated that liposomal formulation FII containing drug/DSPC/cholesterol in the ratio of 1:2:0.5 showed the highest encapsulation efficiency $(85.8 \pm 7.2 \%)$ as compared to other proliposomal formulations. This difference in entrapment values might be due to the bonding forces between tacrolimus and phospholipid (head group) with 


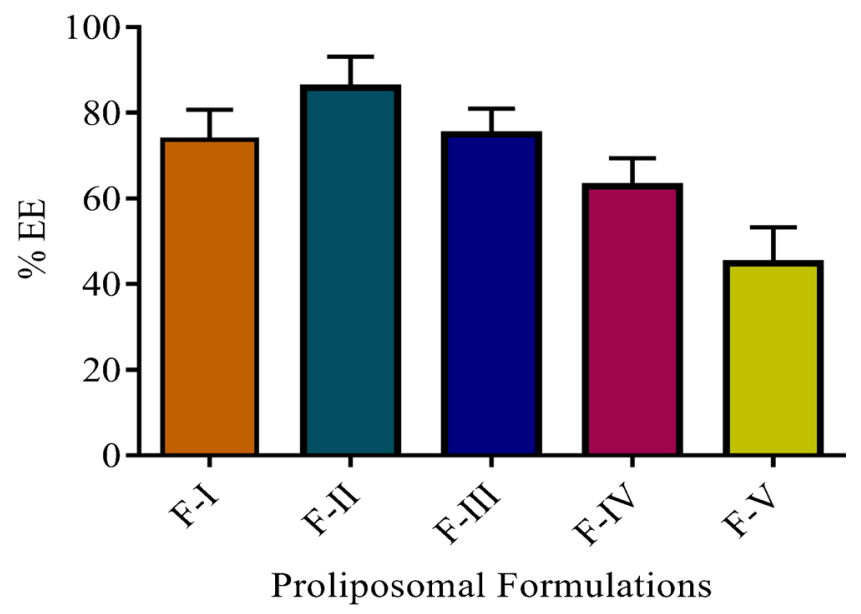

Fig. 1. Encapsulation efficiency of proliposomal formulations

varying carbon chain lengths. The increase in the fatty acid chain length improves the stability of the vesicle membrane and prevents the expulsion of the drug from vesicles leading to greater efficiency (49). Formulation F-V containing drug/DMPG/cholesterol in the ratio of 1:2:0.5 showed the lowest encapsulation efficiency $(44.8 \pm 8.4 \%)$, and this could be due to a weak bilayer structure and decreased packaging space for accommodating the drug molecule which might have resulted in drug leaking (44). It has been reported that proliposomes prepared using DSPC and higher molar ratios of cholesterol demonstrated enhanced stability in acidic $\mathrm{pH}$ (pH 1.2) and bile solution (54). Based on the particle size, zeta potential, encapsulation efficiency, and proliposomal formulation, F-II was selected for further studies.

\section{Formation of Liposomes}

The cryo-TEM images of hydrated proliposomes showed the presence of multilamellar and oligolamellar vesicles in polynomial distribution (Fig. 2a, b) correlating with our results from particle size analysis (Table I).

\section{Differential Scanning Calorimetric Analysis}

The DSC thermograms of pure tacrolimus, proliposomal formulation (F-II), and physical mixture (containing drug, DSPC, and cholesterol) are depicted in Fig. 3. The reported phase transition temperature $\left(T_{\mathrm{c}}\right)$ of DSPC is in the range 54$56^{\circ} \mathrm{C}$. For tacrolimus, the melting transition appears at 128 $132^{\circ} \mathrm{C}$. However, for proliposomal formulation F-II, the endotherm peak was found to be broad with a significant shift in the melting temperature $\left(68-72^{\circ} \mathrm{C}\right)$ when compared to pure tacrolimus and physical mixture, indicating a possible interaction between the DSPC and tacrolimus. This interaction might be an indication of the changes in the solid state of the drug from native crystalline to amorphous or molecular state (1)

\section{Drug Release Study}

The in vitro drug release profiles of pure tacrolimus and proliposomes in purified water are depicted in Fig. 4. After
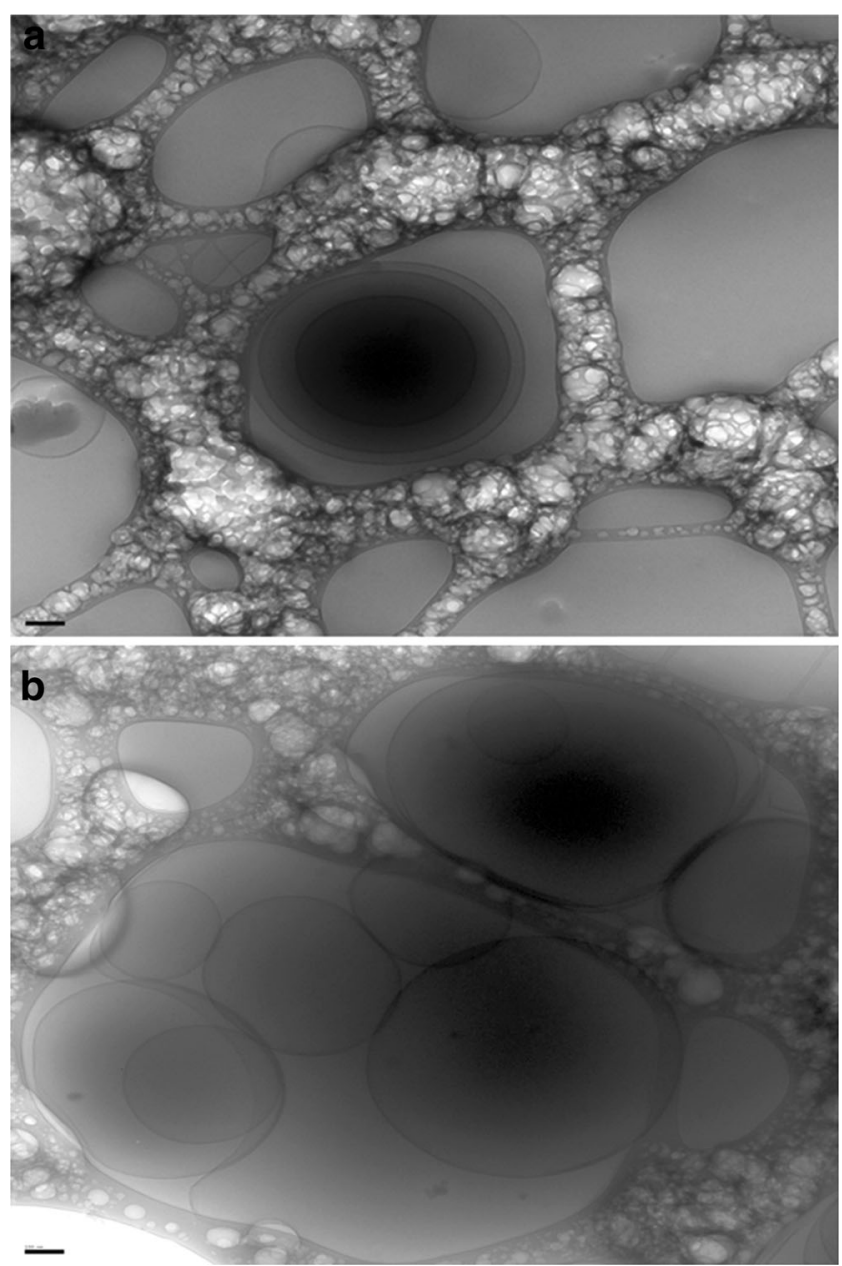

Fig. 2. a Cryo-TEM image showing the multilamellar vesicles of tacrolimus proliposomal formulation (bar=100 nm). b Cryo-TEM image showing the oligolamellar vesicles of tacrolimus proliposomal formulation $($ bar $=100 \mathrm{~nm})$

$60 \mathrm{~min}$, the amount of drug released in purified water from the capsules containing proliposomes was significantly higher as compared to pure drug. The release of the drug from proliposomal formulation was 4.6-fold higher as compared to pure tacrolimus (Fig. 4). The dissolution results were further assessed by analyzing the MDT using ANOVA to study the statistical variance (45). The ANOVA analysis of MDT data of proliposomes $\left(P<0.05, F_{\text {critical }}=5.3\right.$ and $F_{\text {calculated }}=7.5$ ) demonstrated a significant enhancement in drug release compared to pure tacrolimus. The enhanced dissolution with proliposomes may be attributed to the presence of DSPC in the composition or due to change in the solid-state property of the drug (55). The similarity factor $\left(f_{2}\right)$ estimated on the dissolution data of proliposomes versus pure tacrolimus was 11.06 in purified water indicating dissimilarity between the formulations. Due to the poor solubility of tacrolimus in purified water, dissolution studies were performed in water to discriminate the dissolution of tacrolimus from different formulations. In our study, using proliposomes, we have demonstrated that the dissolution of tacrolimus can be significantly improved in purified water within $1 \mathrm{~h}$ $(P<0.05)$. 


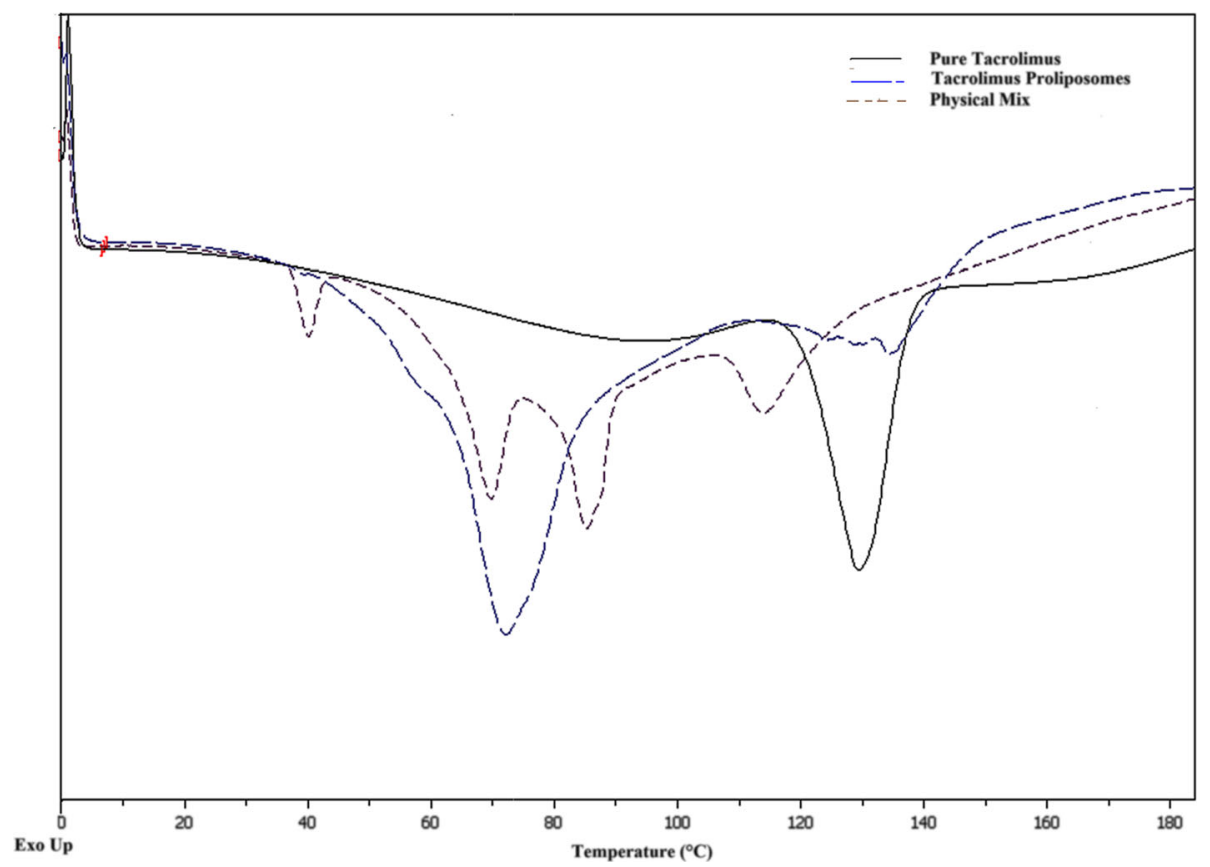

Fig. 3. DSC thermograms of pure tacrolimus, physical mixture (drug, DSPC, and cholesterol), and proliposomal formulation (F-II)

\section{Parallel Artificial Membrane Permeability Assay Studies}

PAMPA is an in vitro drug permeability model (noncell based) that gives information on permeability of drug through passive transcellular mechanism. The PAMPA model lacks pores and transporters for drug permeability and a $\log P$ (lipophilicity) of higher value $(>3)$ is a prerequisite for passive diffusion of drug through the membranes. The effect of proliposomes on tacrolimus permeability in the PAMPA model is depicted in Fig. 5. The results show enhanced permeability of proliposomes indicating improved passive permeation of drug across the simulated membrane barrier. The effective permeability $\left(P_{\mathrm{e}}\right)$ values for the pure drug and proliposomal formulation was found to be $0.9 \times 10^{-5}$ and $1.9 \times 10^{-5} \mathrm{~cm} / \mathrm{s}$, respectively, which indicates that liposomes have shown

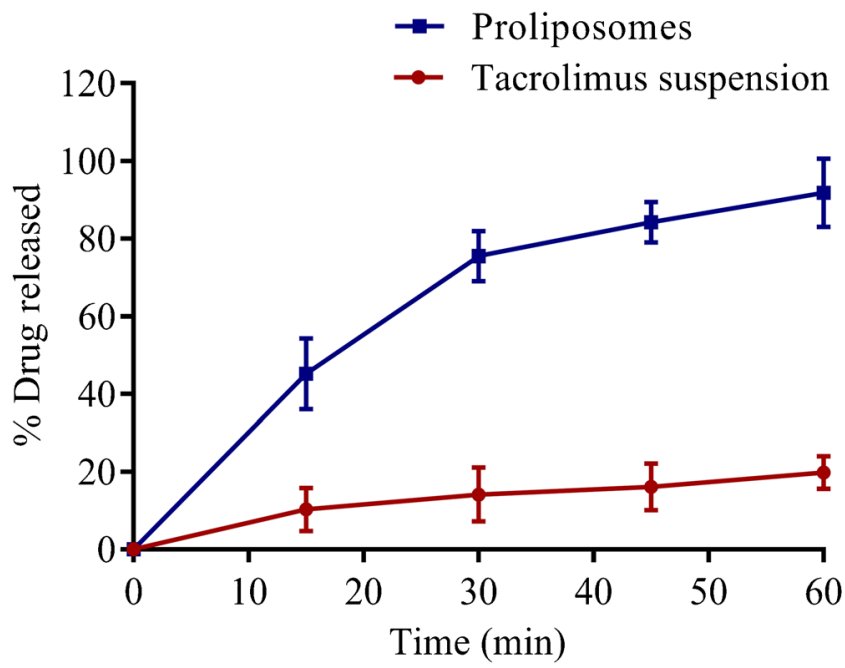

Fig. 4. Comparative dissolution profile of pure tacrolimus and proliposomes in purified water $(n=3$, mean \pm SD) higher permeability when compared to pure tacrolimus (Fig. 5). The ANOVA results further demonstrated that proliposomes $\left(P<0.05, F_{\text {critical }}=7.7\right.$ and $\left.F_{\text {calculated }}=150.1\right)$ significantly enhanced the permeability in comparison to pure tacrolimus. The increased permeability of proliposomes may be attributed to encapsulation of the drug in the lipid core which might have resulted in improved passive diffusion (56).

\section{Everted Rat Intestinal Perfusion Studies}

In order to confirm the data obtained from in vitro permeation studies, an ex vivo rat intestinal perfusion model was performed. The studies indicated a 4.8 -fold increase in the transport with proliposomal formulation against the pure drug (Fig. 6). ANOVA analysis indicated that proliposomes $\left(P<0.05, F_{\text {critical }}=4.9\right.$ and $\left.F_{\text {calculated }}=12.9\right)$ significantly in-

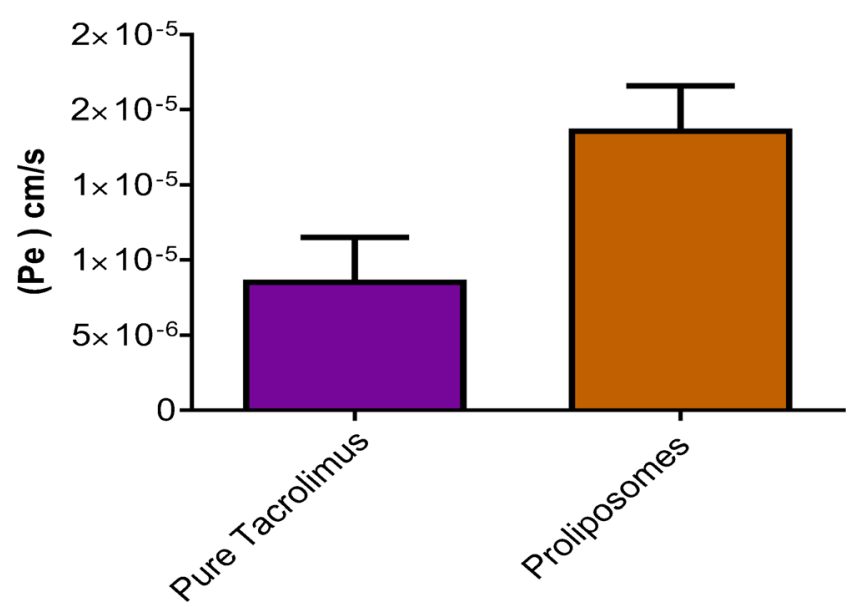

Fig. 5. PAMPA membrane transport profile of tacrolimus from pure drug suspension and proliposomes $(n=3$, mean \pm SD) 


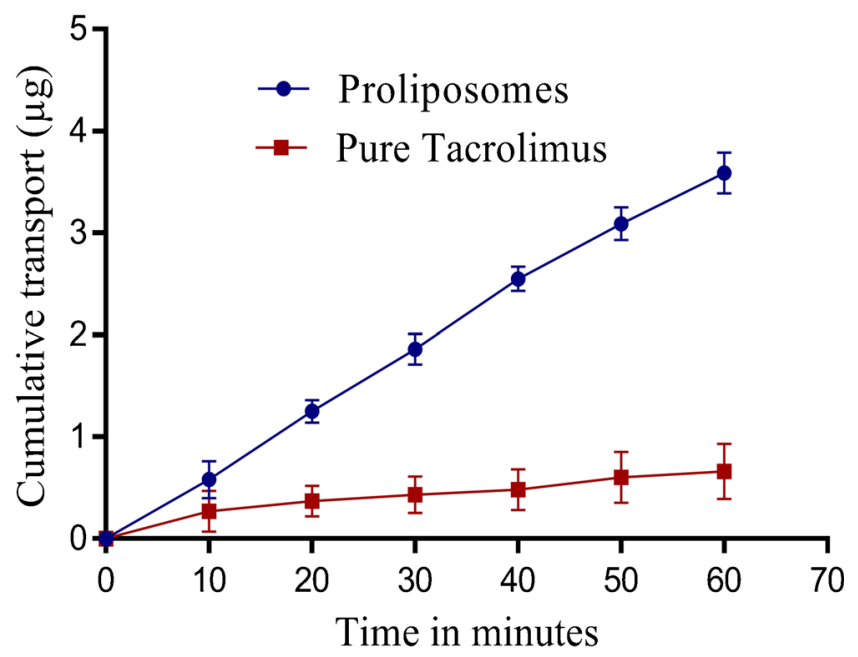

Fig. 6. Everted rat intestinal perfusion profile of tacrolimus from pure drug suspension and proliposomes $(n=3$, mean $\pm \mathrm{SD})$

creased the permeability compared to pure drug. Drug permeability across the intestine is the determining factor for overall absorption of the drug administered orally. The estimation of drug transport using everted rat intestine has been extensively studied and reported to be an excellent model for predicting the drug transport across the intestinal membrane (57).

The everted rat intestinal perfusion studies showed that the amount of drug perfused across the serosal membrane increased exponentially over time as compared to pure tacrolimus. The increased concentration across serosal membrane clearly indicates that the proliposomal formulation showed improved passive diffusion as compared to pure tacrolimus. The increased permeation of the drug with the proliposomes may be due to the increased dissolution of the drug to permeate across the membrane.

The $P_{\text {eff }}, K_{\mathrm{a}}$, and ER of pure drug and proliposomes were estimated and the results were summarized in Table II. The estimated $P_{\text {eff }}$ values for pure drug and proliposomes were $3.20 \pm 0.25 \times 10^{-6}$ and $5.15 \pm 0.43 \times 10^{-6} \mathrm{~cm} / \mathrm{s}$, respectively. The higher $P_{\text {eff }}$ for tacrolimus from proliposomes in comparison to pure drug demonstrated the potential for overcoming the membrane barriers of the GI tract and facilitating the absorption of tacrolimus. The rate of absorption, $K_{\mathrm{a}}$, for proliposomes was 4.2-fold higher compared to the pure tacrolimus. An ER value greater than 1 generally indicates an improved permeation (44), and in our investigation, proliposomal formulation has an ER value higher than 1 which indicates the role of proliposomes as carriers for improving the oral bioavailability of drugs with poor solubility.

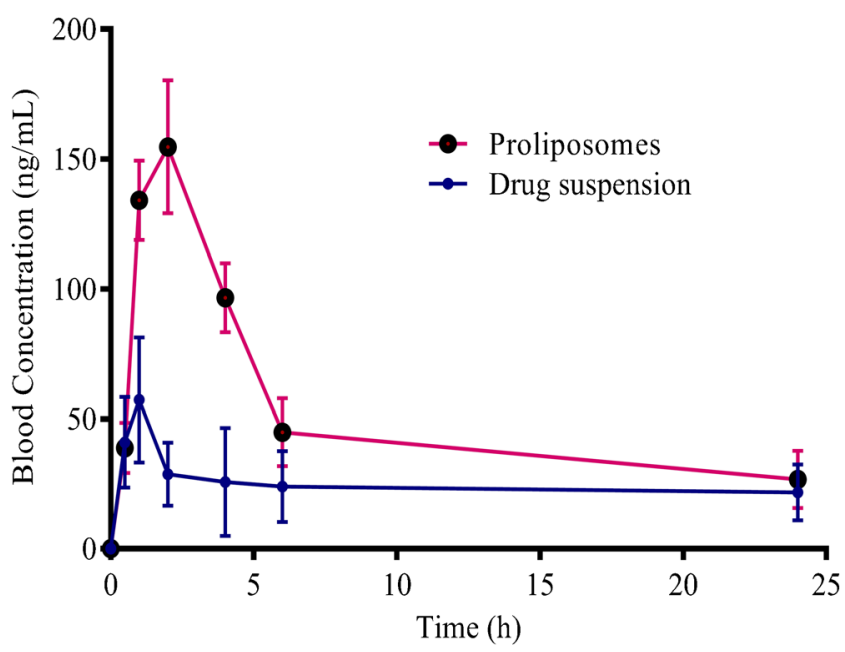

Fig. 7. Mean plasma concentrations of tacrolimus following oral administration of pure drug suspension and proliposomes to male SD rats $(n=5$, mean $\pm \mathrm{SD})$

PAMPA provides an estimate transcellular diffusion of the drug across lipid bilayers (58) and is considered to be a passive absorption model since it is an artificially prepared membrane and does not contain cellular tight junctions and transporters, whereas the rat intestinal perfusion model comprises various influx and efflux transporters or paracellular pores for effective transportation of the drug across the membranes. In vitro transport studies in PAMPA and everted rat intestinal perfusion models revealed that liposomal formulation showed increased permeability of tacrolimus. The enhanced in vitro drug permeability appears to be due to the combined effect of improved dissolution and increased uptake of liposomes through intact cell membranes. It has been reported that small polar molecules are permeated across the intestinal barriers predominantly through the paracellular pathway (59). The transport of drugs from this route is controlled by the tight cell junctions; therefore, widening of these tight junctions may provide a path to improve paracellular transport (60). Cholesterol and bile salts are reported to facilitate the uptake of drugs transported through the transcellular route (61).

\section{Pharmacokinetic Study}

Formulating tacrolimus into proliposomes resulted in an improved in vitro dissolution and in vitro permeation. To confirm these findings, in vivo pharmacokinetic studies were conducted in male SD rats. The mean plasma concentration versus time profiles of tacrolimus after oral administration of

Table II. In Situ Parameters of Tacrolimus from Pure Drug Suspension and Proliposomal Formulations Across the Rat Intestine (Mean \pm SD; $n=3)$

\begin{tabular}{lcc}
\hline Formulation & $P_{\text {eff }}(\mathrm{cm} / \mathrm{s}) \times 10^{-6}($ mean $\pm \mathrm{SD})$ & $K_{\mathrm{a}}\left(\mathrm{h}^{-1}\right)(\mathrm{mean} \pm \mathrm{SD})$ \\
\hline Pure drug suspension & $3.20 \pm 0.25$ & $0.017 \pm 0.002$ \\
Proliposomes & $5.15 \pm 0.43$ & $0.071 \pm 0.004$ \\
\hline
\end{tabular}

$P_{\text {eff }}$ effective permeability coefficient, $K_{a}$ absorption rate constant, $E R$ enhancement ratio 
Table III. Pharmacokinetic Parameters of Tacrolimus Suspension and Proliposomal Formulations (Mean \pm SD; $n=5$ )

\begin{tabular}{lll}
\hline PK parameters & Pure drug suspension (mean \pm SD) & Proliposomal Formulation $(\mathrm{mean} \pm \mathrm{SD})$ \\
\hline$T_{\max }(\mathrm{h})$ & $0.75 \pm 0.12$ & $2.10 \pm 0.68$ \\
$C_{\max }(\mathrm{ng} / \mathrm{mL})$ & $57.02 \pm 0.25$ & $154.75 \pm 31.04$ \\
$\mathrm{AUC}_{0-24}(\mathrm{ng} / \mathrm{mL} / \mathrm{h})$ & $601.63 \pm 92.42$ & $1163.14 \pm 221.78$ \\
$t_{1 / 2}(\mathrm{~h})$ & $26.90 \pm 2.42$ & $17.33 \pm 3.18$ \\
Relative bioavailability $^{a}(\%)$ & - & 193.33 \\
\hline
\end{tabular}

$P K$ pharmacokinetic, $T_{\max }$ time to maximum plasma concentration, $C_{\max }$ maximum concentration, $A U C$ area under the curve, $t_{1 / 2}$ half-life aRelative bioavailability $=\left[\mathrm{AUC}_{\text {proliposomes }}\right] /\left[\mathrm{AUC}_{\text {pure drug suspension }}\right] * 100$

proliposomes and pure drug suspension at a dose of $10 \mathrm{mg} / \mathrm{kg}$ are depicted in Fig. 7. The pharmacokinetic parameters obtained from the study are summarized in Table III. The results indicate that $T_{\max }$ from pure tacrolimus formulation was 0.75 $\pm 0.12 \mathrm{~h}$, whereas for proliposomes, it was $2.10 \pm 0.68 \mathrm{~h}$. The $C_{\text {max }}$ of proliposomes was 2.50 -fold higher as compared to pure tacrolimus. The $t_{1 / 2}$ of pure tacrolimus and proliposomal formulations was $26.90 \pm 2.42$ and $17.33 \pm 3.18$, respectively. The decrease in $t_{1 / 2}$ of proliposomal formulation indicates less accumulation of the drug that may result in reduced toxicity compared to pure tacrolimus. The systemic exposure $\left(\mathrm{AUC}_{0}\right.$ 24) of proliposomes and pure tacrolimus suspension was $601.63 \pm 92.42$ and $1163.14 \pm 221.78 \mathrm{ng} / \mathrm{mL} / \mathrm{h}$, respectively, showing nearly a 2 -fold increase for proliposomes in comparison to pure tacrolimus suspension. This increase in the overall exposure of the proliposomal formulation correlates with the increase in their relative bioavailability, which was $193.33 \%$.

The increased $C_{\max }$ observed with proliposomal formulation further substantiates the in vitro and ex vivo findings. The delay in $T_{\max }$ with the proliposomal formulation $(2.10 \mathrm{~h})$ when compared to pure tacrolimus $(<1 \mathrm{~h})$ may be attributed to the time taken for the release of the drug from the multilamellar or oligolamellar liposomes. The increased $\mathrm{AUC}_{0-24}$ by proliposomes indicates improved absorption of tacrolimus from the GI. The in vivo pharmacokinetic results confirm our in vitro findings that by formulating proliposomes, we can deliver the drug in a well-dispersed form in the upper GI, to enable rapid absorption of the drug from the site. The in vivo pharmacokinetic results revealed that there is a potential for improving the dissolution of tacrolimus by formulating as proliposomes, thereby enhancing the transportation across the intestinal membrane. In addition, the increased lipophilicity of the encapsulated drug may also further influence the absorption of the drug in vivo.
The improved pharmacokinetic performance of tacrolimus proliposomes may be attributed to the enhanced absorption of tacrolimus from the liposomes. In addition, lymphatic uptake of the drug bypassing the first-pass metabolism in the GI might also have resulted in increased bioavailability. The study results indicate that by designing an optimal formulation, the oral bioavailability of tacrolimus can be improved which may further translate into reduced dose and doserelated toxicity.

\section{Formulation Stability}

The stability results of the selected proliposomal formulation stored at room temperature $\left(25^{\circ} \mathrm{C}\right)$ and refrigerated $(5 \pm$ $3^{\circ} \mathrm{C}$ ) conditions are summarized in Table IV. There were no significant changes observed in the particle size distribution and drug content values indicating the stability of the developed formulation on storage at room temperature and refrigerated conditions.

\section{CONCLUSIONS}

The present investigation reports the development of proliposomal formulation for a poorly bioavailable drug, tacrolimus. The developed formulation showed a significant increase in drug release as compared to the pure tacrolimus under the same conditions. The in vitro transport studies in PAMPA and everted rat intestine perfusion models revealed a significant enhancement in drug permeation. The in vivo pharmacokinetic results are promising and convincing in demonstrating the improved oral bioavailability of tacrolimus using proliposomes. Further, studies are warranted to establish the proof of concept and clinical relevance of the developed formulation.

Table IV. Stability Results of Proliposomal Formulations

\begin{tabular}{|c|c|c|c|c|}
\hline \multirow[t]{3}{*}{ Time (months) } & \multicolumn{4}{|l|}{ Proliposomes } \\
\hline & \multicolumn{2}{|c|}{ Particle size $(\mathrm{nm})$} & \multicolumn{2}{|l|}{ Assay (\%) } \\
\hline & $25^{\circ} \mathrm{C} / 60 \% \mathrm{RH}$ & $5 \pm 3^{\circ} \mathrm{C}$ & $25^{\circ} \mathrm{C} / 60 \% \mathrm{RH}$ & $5 \pm 3^{\circ} \mathrm{C}$ \\
\hline Initial & $858.2 \pm 49.1$ & & $98.6 \pm 2.1$ & \\
\hline 0.5 & $886.1 \pm 72.8$ & $882.4 \pm 72.2$ & $98.6 \pm 1.6$ & $98.1 \pm 1.9$ \\
\hline 1 & $894.7 \pm 81.3$ & $891.4 \pm 52.1$ & $97.2 \pm 2.8$ & $97.1 \pm 1.1$ \\
\hline 3 & $906.5 \pm 68.2$ & $896.4 \pm 62.4$ & $96.1 \pm 1.3$ & $98.1 \pm 1.5$ \\
\hline
\end{tabular}

$R H$ relative humidity 


\section{ACKNOWLEDGMENTS}

The authors express their sincere gratitude to Western University of Health Sciences, Pomona, California for awarding postdoctoral fellowship to carry out this project.

\section{Compliance with Ethical Standards}

Conflict of Interest The authors declare that they have no competing interests.

\section{REFERENCES}

1. Bobbala SKR, Veerareddy PR. Formulation, evaluation, and pharmacokinetics of isradipine proliposomes for oral delivery. J Liposome Res. 2012;22(4):285-94.

2. Hiremath PS, Soppimath KS, Betageri GV. Proliposomes of exemestane for improved oral delivery: formulation and in vitro evaluation using PAMPA, Caco-2 and rat intestine. Int J Pharm. 2009;380(1):96-104

3. Katare O, Vyas S, Dixit V. Effervescent granule based proliposomes of ibuprofen. J Microencapsul. 1990;7(4):455-62.

4. Katare O, Vyas S, Dixit V. Proliposomes of indomethacin for oral administration. J Microencapsul. 1991;8(1):1-7.

5. Potluri P, Betageri GV. Mixed-micellar proliposomal systems for enhanced oral delivery of progesterone. Drug Deliv. 2006;13(3):227-32.

6. Basalious EB, Shawky N, Badr-Eldin SM. SNEDDS containing bioenhancers for improvement of dissolution and oral absorption of lacidipine. I: development and optimization. Int J Pharm. 2010;391(1):203-11.

7. Parmar N, Singla N, Amin S, Kohli K. Study of cosurfactant effect on nanoemulsifying area and development of lercanidipine loaded (SNEDDS) self nanoemulsifying drug delivery system. Colloids Surf B: Biointerfaces. 2011;86(2):327-38.

8. Kalepu S, Nekkanti V. Insoluble drug delivery strategies: review of recent advances and business prospects. Acta Pharm Sin B. 2015.

9. Aungst BJ, Saitoh H, Burcham DL, Huang S-M, Mousa SA, Hussain MA. Enhancement of the intestinal absorption of peptides and nonpeptides. J Control Release. 1996;41(1):19-31.

10. Nekkanti V, Venkatesan N, Betageri GV. Proliposomes for oral delivery: progress and challenges. Curr Pharm Biotechnol. 2014;16(4):303-12.

11. Nekkanti V, Venkatesan N, Wang Z, Betageri GV. Improved oral bioavailability of valsartan using proliposomes: design, characterization and in vivo pharmacokinetics. Drug Dev Ind Pharm. 2015;1-12.

12. Wang Y-p, Gan Y, X-x Z. Novel gastroretentive sustainedrelease tablet of tacrolimus based on self-microemulsifying mixture: in vitro evaluation and in vivo bioavailability test. Acta Pharmacol Sin. 2011;32(10):1294-302.

13. Patel P, Patel H, Panchal S, Mehta T. Formulation strategies for drug delivery of tacrolimus: an overview. Int J Pharm Investig. 2012;2(4):169.

14. Saeki T, Ueda K, Tanigawara Y, Hori R, Komano T. Human Pglycoprotein transports cyclosporin A and FK506. J Biol Chem. 1993;268(9):6077-80.

15. Roy JN, Barama A, Poirier C, Vinet B, Roger M. Cyp3A4, Cyp3A5, and MDR-1 genetic influences on tacrolimus pharmacokinetics in renal transplant recipients. Pharmacogenet Genomics. 2006;16(9):659-65.

16. Shapiro R, editor. Tacrolimus in solid organ transplantation: an update. Transplantation proceedings; 1999: Elsevier.

17. Staatz CE, Tett SE. Clinical pharmacokinetics and pharmacodynamics of tacrolimus in solid organ transplantation. Clin Pharmacokinet. 2004;43(10):623-53.

18. Venkataramanan R, Swaminathan A, Prasad T, Jain A, Zuckerman S, Warty V, et al. Clinical pharmacokinetics of tacrolimus. Clin Pharmacokinet. 1995;29(6):404-30.

19. Kershner RP, Fitzsimmons WE. Relationship of FK506 whole blood concentrations and efficacy and toxicity after liver and kidney transplantation. Transplantation. 1996;62(7):920-6.
20. Jeong H, Chiou W. Role of P-glycoprotein in the hepatic metabolism of tacrolimus. Xenobiotica. 2006;36(1):1-13.

21. Yura H, Yoshimura N, Hamashima T, Akamatsu K, Nishikawa M, Takakura Y, et al. Synthesis and pharmacokinetics of a novel macromolecular prodrug of Tacrolimus (FK506), FK506-dextran conjugate. J Control Release. 1999;57(1):87-99.

22. Arima H, Yunomae K, Miyake K, Irie T, Hirayama F, Uekama K. Comparative studies of the enhancing effects of cyclodextrins on the solubility and oral bioavailability of tacrolimus in rats. J Pharm Sci. 2001;90(6):690-701.

23. Yamashita K, Nakate T, Okimoto K, Ohike A, Tokunaga Y, Ibuki $\mathrm{R}$, et al. Establishment of new preparation method for solid dispersion formulation of tacrolimus. Int $\mathrm{J}$ Pharm. 2003;267(1):79-91.

24. Kagayama A, Tanimoto S, Fujisaki J, Kaibara A, Ohara K, Iwasaki K, et al. Oral absorption of FK506 in rats. Pharm Res. 1993;10(10):1446-50.

25. McAlister V, Keshavamurthy M, Lee T, editors. Oral delivery of liposomal tacrolimus: increased efficacy and reduced toxicity. Transplantation proceedings; 1999: Elsevier.

26. Borhade V, Nair H, Hegde D. Design and evaluation of selfmicroemulsifying drug delivery system (SMEDDS) of tacrolimus. AAPS PharmSciTech. 2008;9(1):13-21.

27. Wang Y, Sun J, Zhang T, Liu H, He F, He Z. Enhanced oral bioavailability of tacrolimus in rats by self-microemulsifying drug delivery systems. Drug Dev Ind Pharm. 2011;37(10):1225-30.

28. Dixit AR, Rajput SJ, Patel SG. Preparation and bioavailability assessment of SMEDDS containing valsartan. AAPS PharmSciTech. 2010;11(1):314-21.

29. Nassar T, Rom A, Nyska A, Benita S. A novel nanocapsule delivery system to overcome intestinal degradation and drug transport limited absorption of P-glycoprotein substrate drugs. Pharm Res. 2008;25(9):2019-29.

30. Wang Y, Wang C, Fu S, Liu Q, Dou D, Lv H, et al. Preparation of tacrolimus loaded micelles based on poly ( $\varepsilon$-caprolactone)-poly (ethylene glycol)-poly ( $\varepsilon$-caprolactone). Int J Pharm. 2011;407(1):184-9.

31. Ali SM, Ahmad A, Sheikh S, Ahmad MU, Rane RC, Kale P, et al. Polyoxyl 60 hydrogenated castor oil free nanosomal formulation of immunosuppressant tacrolimus: pharmacokinetics, safety, and tolerability in rodents and humans. Int Immunopharmacol. 2010;10(3):325-30.

32. Lamprecht A, Yamamoto H, Takeuchi H, Kawashima Y. A $\mathrm{pH}$-sensitive microsphere system for the colon delivery of tacrolimus containing nanoparticles. J Control Release. 2005;104(2):337-46.

33. Chougule M, Padhi B, Misra A. Nano-liposomal dry powder inhaler of tacrolimus: preparation, characterization, and pulmonary pharmacokinetics. Int J Nanomedicine. 2007;2(4):675.

34. Cornaire G, Woodley J, Hermann P, Cloarec A, Arellano C, Houin G. Impact of excipients on the absorption of $\mathrm{P}$ glycoprotein substrates in vitro and in vivo. Int $\mathrm{J}$ Pharm. 2004;278(1):119-31.

35. Porter CJ, Charman WN. Intestinal lymphatic drug transport: an update. Adv Drug Deliv Rev. 2001;50(1):61-80.

36. Charman WN. Lipid vehicle and formulation effects on intestinal lymphatic drug transport. Lymphatic transport of drugs. Boca Raton: CRC; 1992. p. 113-79.

37. $\mathrm{Xu} \mathrm{H}$, He L, Nie S, Guan J, Zhang X, Yang X, et al. Optimized preparation of vinpocetine proliposomes by a novel method and in vivo evaluation of its pharmacokinetics in New Zealand rabbits. J Control Release. 2009;140(1):61-8.

38. Chu C, Tong S-s, Xu Y, Wang L, Fu M, Ge Y-r, et al. Proliposomes for oral delivery of dehydrosilymarin: preparation and evaluation in vitro and in vivo. Acta Pharmacol Sin. 2011;32(7):973-80.

39. Janga KY, Jukanti R, Velpula A, Sunkavalli S, Bandari S, Kandadi $\mathrm{P}$, et al. Bioavailability enhancement of zaleplon via proliposomes: role of surface charge. Eur J Pharm Biopharm. 2012;80(2):347-57.

40. Aburahma MH, Abdelbary GA. Novel diphenyl dimethyl bicarboxylate provesicular powders with enhanced hepatocurative activity: preparation, optimization, in vitro/in vivo 
evaluation. Int J Pharm. 2012;422(1-2):139-50. doi:10.1016/ j.ijpharm.2011.10.043.

41. Ravis WR, Feldman S. Effect of enzyme-inducing and enzymeinhibiting agents on drug absorption I: 3-O-methylglucose transport in rats. J Pharm Sci. 1978;67(2):245-8.

42. Barthe L, Woodley J, Houin G. Gastrointestinal absorption of drugs: methods and studies. Fundam Clin Pharmacol. 1999;13(2):154-68.

43. Issa C, Gupta P, Bansal AK. Implications of density correction in gravimetric method for water flux determination using rat singlepass intestinal perfusion technique: a technical note. AAPS PharmSciTech. 2003;4(2):44-9.

44. Jukanti R, Mateti A, Bandari S, Veerareddy PR. Transdermal delivery of acyclovir sodium via carbopol gels: role of chemical permeation enhancers. Lett Drug Des Discov. 2011;8(4):381-9.

45. Costa P, Lobo JMS. Modeling and comparison of dissolution profiles. Eur J Pharm Sci. 2001;13(2):123-33.

46. Shah VP, Tsong Y, Sathe P, Liu J-P. In vitro dissolution profile comparison-statistics and analysis of the similarity factor, $\mathrm{f} 2$. Pharm Res. 1998;15(6):889-96.

47. Payne NI, Timmins P, Ambrose CV, Ward MD, Ridgway F. Proliposomes: a novel solution to an old problem. J Pharm Sci. 1986;75(4):325-9.

48. Gregoriadis G. Engineering liposomes for drug delivery: progress and problems. Trends Biotechnol. 1995;13(12):527-37.

49. Betageri G. Liposomal encapsulation and stability of dideoxyinosine triphosphate. Drug Dev Ind Pharm. 1993;19(5):531-9.

50. Charman WN, Stella VJ, editors. Lymphatic transport of drugs. Boca Raton: CRC; 1992.

51. Elorza M, Garcia dCF, San Juan R, Marcilla A, Rico H, Mormeneo S, et al. Linkages between macromolecules in Candida albicans cell wall. Arch Med Res. 1992;24(3):305-10.
52. Patel J, Patel A, Raval M, Sheth N. Formulation and development of a self-nanoemulsifying drug delivery system of irbesartan. J Adv Pharm Technol Res. 2011;2(1):9.

53. Müller R, Jacobs C, Kayser O. Nanosuspensions as particulate drug formulations in therapy: rationale for development and what we can expect for the future. Adv Drug Deliv Rev. 2001;47(1):3-19.

54. Aramaki Y, Tomizawa H, Hara T, Yachi K, Kikuchi H, Tsuchiya S. Stability of liposomes in vitro and their uptake by rat Peyer's patches following oral administration. Pharm Res. 1993;10(8):1228-31.

55. Mirza S, Miroshnyk I, Habib MJ, Brausch JF, Hussain MD. Enhanced dissolution and oral bioavailability of piroxicam formulations: modulating effect of phospholipids. Pharmaceutics. 2010;2(4):339-50.

56. KV S, Devi GS, Mathew ST. Liposomal formulations of serratiopeptidase: in vitro studies using PAMPA and Caco-2 models. Mol Pharm. 2007;5(1):92-7.

57. Amidon GL, Sinko PJ, Fleisher D. Estimating human oral fraction dose absorbed: a correlation using rat intestinal membrane permeability for passive and carrier-mediated compounds. Pharm Res. 1988;5(10):651-4.

58. Kansy M, Senner F, Gubernator K. Physicochemical high throughput screening: parallel artificial membrane permeation assay in the description of passive absorption processes. J Med Chem. 1998;41(7):1007-10.

59. Artursson P, Palm K, Luthman K. Caco-2 monolayers in experimental and theoretical predictions of drug transport. Adv Drug Deliv Rev. 2001;46(1):27-43.

60. Hochman J, Artursson P. Mechanisms of absorption enhancement and tight junction regulation. J Control Release. 1994;29(3):253-67.

61. Werner U, Kissel T, Reers M. Effects of permeation enhancers on the transport of a peptidomimetic thrombin inhibitor (CRC 220) in a human intestinal cell line (Caco-2). Pharm Res. 1996;13(8):1219-27. 